\title{
A multi-resolution model for the simulation of transient heat and mass transfer
}

\author{
Jahrul M Alam ${ }^{\mathrm{a} *}$, Nicholas K. -R. Kevlahan ${ }^{\mathrm{b}}$, Oleg V. Vasilyev ${ }^{\mathrm{c}}$, and Zahangir Hossain ${ }^{\mathrm{a}}$,

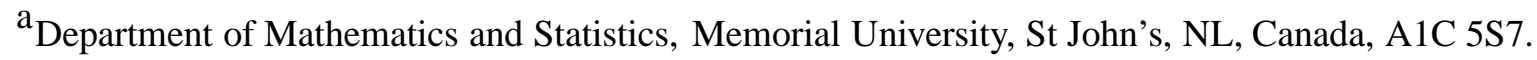

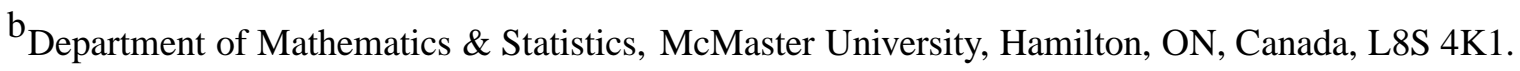 \\ ${ }^{c}$ Department of Mechanical Engineering, University of Colorado, Boulder, CO 80309-0427, USA.
}

\begin{abstract}
The development of an efficient computational methodology for transient heat and mass transfer applications is challenging. When a solution is localized on the fraction of a computational domain, an appropriate adaptive mesh method could minimize computational work. In this paper, we propose a novel adaptive-mesh multi-resolution algorithm for the transient momentum and energy equations. The nonlinear dynamics between the velocity and temperature fields is modeled by solving the coupled system of equations simultaneously, where the rate of convergence has been optimized so that computational cost remains proportional to the number of grid points. Numerical experiments have exhibited good agreements with benchmark simulation data.
\end{abstract}

\footnotetext{
*Corresponding author's email address: alamj@mun.ca.
} 


\begin{tabular}{|c|c|c|c|}
\hline \multicolumn{4}{|c|}{ NOMENCLATURE } \\
\hline$\beta$ & thermal expansion coefficient & $\mathcal{L}$ & nonlinear system of equations \\
\hline$\Delta t$ & time step & $L$ & length scale \\
\hline$\epsilon$ & tolerance & $N$ & number of non-adaptive grid points \\
\hline$\theta_{0}$ & reference temperature & $\mathcal{N}$ & number of adaptive grid points \\
\hline$\theta$ & temperature & $\operatorname{Pr}$ & Prandtl number \\
\hline$\kappa$ & thermal diffusivity & $P$ & pressure \\
\hline$\sigma$ & reference to a wavelet family & $R e$ & Reynolds number \\
\hline \multirow[t]{2}{*}{$\alpha_{k}$} & step length for & $R a$ & Rayleigh number \\
\hline & the relaxation scheme & & \\
\hline$\nu$ & kinematic viscosity & $T_{R}$ & temperature scale \\
\hline$m$ & dimension of a Krylov space & $t$ & dimensionless time \\
\hline$\rho$ & density & $U$ & velocity scale \\
\hline$u$ & dimensionless velocity vector & $(u, v)$ & velocity components \\
\hline $\mathcal{G}^{j}$ & a grid at level $j$ & $\boldsymbol{x}$ & vector form $\boldsymbol{x}=(x, y)$ \\
\hline$g$ & accelaration due to gravity & $(x, y)$ & coordinates \\
\hline$f$ & source term for a PDE & f & a nonlinear algebraic system \\
\hline $\mathcal{J}$ & Jacobian of a nonlinear system & $\mathcal{X}$ & length of the domain in $x$-direction \\
\hline $\mathcal{K}_{m}$ & Krylov space of dimension $m$ & $\mathcal{Y}$ & length of the domain in $y$-direction \\
\hline \multicolumn{4}{|c|}{ Superscripts } \\
\hline$n$ & discrete time level, $n \Delta t$ & $n+1$ & discrete time level, $(n+1) \Delta t$ \\
\hline$k$ & local iteration & $j$ & level of resolution \\
\hline \multicolumn{4}{|c|}{ Subscripts } \\
\hline 2 & vector norm & $\max$ & maximum norm \\
\hline
\end{tabular}

\section{Introduction}

Transient simulations of heat transfer problems are often used to advance scientific knowledge in a wide variety of applications such as the design of thermal systems $[1 ; 2 ; 3]$, material processing [4; 5; 6], biomedical studies [7], fire protection [8], porous media [9], and weather forecasting [10] - e.g. see [11] for a comprehensive survey of recently published works. Toward this direction, recent interests include the development of powerful numerical methods $[12 ; 13 ; 14 ; 15]$ 
so that accuracy of a numerical model is greatly improved without overburdening the computational cost $[16 ; 17 ; 18]$. More specifically, the natural convection and the shear driven circulation in a bounded domain appear frequently in many complex industrial applications such as nuclear reactor insulation, ventilation of rooms, solar energy collection etc. [e.g., see, 19]. The numerical investigation of such convective circulations often requires a high spatial and temporal resolution because the solution contains localized or intermittent structures, or sharp local variations, in which locations of these structures may also vary with time. For such a transient flow simulation, using modern high performance computing (HPC) facilities, one may be able to employ a mesh with extreme high resolution, and there exists a number of commercial or freely available Computational Fluid Dynamics (CFD) softwares that may be used for this purpose. Alternatively, using an adhoc error indicator, an adaptive mesh refinement (AMR) approach was introduced in $[20 ; 21 ; 22]$, which can be used to improve the accuracy of a transient simulation. In [23], such an adaptive mesh method was studied for transient heat transfer applications. In [24], the use of interpolating wavelet transform was studied so that a spatial mesh can be refined locally at each time step without using any ad-hoc error indicator.

There are two principal drawbacks. First, for a $d$-dimensional non-adaptive mesh, if the resolution increases by a factor of 2 in each direction, the total number of grid points $N$ increases by a factor of $2^{d}$ if the mesh is refined uniformly. Clearly, this approach increases drastically both the CPU time and the memory. Second, using a locally refined high resolution adaptive mesh, one may be able to optimize the number of grid points $\mathcal{N}$ on an adaptive mesh such that $\mathcal{N} \ll N$. However, this approach would require an extremely small time step for using an explicit time integration scheme because of the Courant-Friedrichs-Lewy (CFL) criterion [23]. Moreover, a fully implicit method for both the linear and the nonlinear terms of momentum and energy equations requires matrix-vector multiplications at each time step due to linearization of the nonlinear system, which has a computational cost that is proportional to $\mathcal{O}\left(\mathcal{N}^{2}\right)$ (e.g. see, [25]). Therefore, the development of a more powerful numerical method is essential for an optimal use of adaptive mesh methods for transient simulations of heat transfer applications. Ref [18] used a non-adaptive mesh to study other aspects of cost effectiveness, such as the pressure-based discretization, for heat and mass transfer applications. Generally speaking, the nonlinear dynamics of the CFD or heat transfer problems is an everlasting computational challenge, and advanced methodologies such as adaptive mesh and multi-scale solvers can be used to improve the performance of a CFD model for heat transfer application. 
In this research, we study the development of a novel approach - the adaptive multi-resolution methodology (AMR) - for the solution of nonlinear, advection dominated, thermally or shear driven transient flow problems. We aim to put together benefits of three powerful techniques that have been evolved independently to the field of CFD. First, to model intermittent spatial features efficiently, an adaptive mesh is constructed from the second-generation wavelet transform of a transient variable, where the spatial discretization is computed with an adaptive wavelet collocation method (AWCM) [26; 27]. Second, to adapt in space and time, and to remove the CFL restriction on time steps, a second-order fully implicit fractional time integration scheme has been studied, where ideas from the full approximation scheme (FAS) are used for solving the simultaneous system of equations iteratively at each time step [28]. Third, some benefits of Jacobian-free NewtonKrylov method $(\mathrm{JFNK})$ are useful to ensure that the computational complexity remains $\mathcal{O}(\mathcal{N})$, where $\mathcal{N}$ is the number of points on the adaptive mesh. Note that we have used $N$ for the number of points on a non-adaptive mesh, and $\mathcal{N}$ for that on an adaptive mesh, where usually $\mathcal{N} \ll N$. We want to develop an adaptive mesh algorithm for simulating a transient problem such that the computational cost increases with $\mathcal{N}$ only linearly if the mesh is refined locally, where the desired accuracy will be achieved according to a given a priori error tolerance. Moreover, we want that $\mathcal{N}$ does not increase linearly if the tolerance for the accuracy measure is reduced; i.e. the accuracy is improved. In such an adaptive computational model, the number of grid points on the adapted mesh indicates the saving of CPU time with respect to calculations on a non-adaptive mesh when the CPU time is linearly proportional to the number of points $\mathcal{N}$. To achieve this goal, instead of combining three powerful methods, e.g., AWCM, FAS, and JFNK directly, we have considered only some benefits of each of these methods to develop a new algorithm - AMR - for heat and mass transfer applications. This paper presents key ingredients of this AMR approach, and verifies its performance with transient numerical simulations in comparison with data available from previously published articles.

The set of equations and temporal integration scheme are presented in section $\S 2$. In section $\S 3$, we outline the basic concepts of wavelet based numerical approximation. The proposed MRA methodology has been presented in $\S 4$. Numerical experiments have been summarized in section $\S 5$, where we verify that the CPU time increases approximately linearly with the number of grid points $\mathcal{N}$ for all examples. Moreover, we have found that $\mathcal{N} \ll N$ in comparision with benchmark data for all numerical experiments. Finally, we have summarized the main results in section $\S 6$. 


\section{Mathematical formulation and temporal integration}

\subsection{Governing equations}

The flow under investigation in this study is governed by the Navier-Stokes equation, and its thermodynamic state is described by $\rho=\rho(p, \theta)$, where the thermodynamic variables are density $(\rho)$, pressure $(p)$, and temperature $(\theta)$. The Boussinesq assumption has been adopted. First, the dependence of density $(\rho)$ on pressure $(p)$ has been neglected; i.e. $\rho \neq \rho(p)$. Secondly, the dependence of density on temperature has been approximated by

$$
\rho(\theta)=\rho\left(\theta_{0}\right)\left[1-\beta\left(\theta-\theta_{0}\right)\right]
$$

where $\beta$ is the coefficient of thermal expansion. The governing system of equations in dimensionless variables include the following PDEs:

$$
\begin{gathered}
\nabla \cdot \boldsymbol{u}=0 \\
\frac{\partial \boldsymbol{u}}{\partial t}+\boldsymbol{u} \cdot \nabla \boldsymbol{u}=-\nabla P+\sqrt{\frac{P r}{R a}} \nabla^{2} \boldsymbol{u}+\gamma \theta \hat{\boldsymbol{k}} \\
\frac{\partial \theta}{\partial t}+\boldsymbol{u} \cdot \nabla \theta=\frac{1}{\sqrt{P r R a}} \nabla^{2} \theta
\end{gathered}
$$

In the above system, characteristic scales for length, velocity, and temperature are $L, U$, and $T_{R}$ respectively. The dimensionless number $\gamma=0$ corresponds to a shear driven flow , and $\gamma=1$ corresponds to a thermally driven flow, where $U=\sqrt{g \beta L T_{R}}$. The Prandtl and Rayleigh numbers are, respectively, defined by

$$
\operatorname{Pr}=\frac{\nu}{\kappa}, \text { and } R a=\frac{g \beta L^{3} T_{R}}{\nu \kappa}
$$

which gives a Reynolds number

$$
R e=\frac{\sqrt{g \beta L^{3} T_{R}}}{\nu}=\frac{U L}{\nu} \text { with } R e^{2}=R a / P r
$$

Scientific journals publish a number of articles based on the system (1-3), which is a fundamental mathematical model for heat and mass transfer applications. The proposed methodology is thus tested by solving these equations.

Let us now present necessary initial and boundary conditions for simulating a shear driven as 
well as a thermally driven circulation in a bounded domain.

\subsubsection{Conditions and parameters for a shear driven flow}

The set of equations (1-2) with $\gamma=0$ governs a shear-driven, incompressible flow in $\bar{\Omega}=\Omega \cup \partial \Omega$, where the temperature equation (3) is excluded from the numerical solution procedure. Here, $\partial \Omega$ is the boundary of the two-dimensional cavity $\Omega=(0, \mathcal{X}) \times(0, \mathcal{Y})$. The initial and boundary conditions are given by

(initial condition)

$$
(u, v)=(0,0) \quad \forall(x, y) \in(0, \mathcal{X}) \times(0, \mathcal{Y}) \text { at } t=0
$$

(boundary conditions)

$(u, v)=(V, 0)$

$\forall x \in[0, \mathcal{X}], y=\mathcal{Y}$, at $t \geq 0$ (top wall),

$(u, v)=(0,0)$

$\forall x \in[0, \mathcal{X}], y=0$, at $t \geq 0$ (bottom wall), and

$(u, v)=(0,0)$

$\forall y \in[0, \mathcal{Y}], x=0, x=\mathcal{X}$, at $t \geq 0$ (side walls).

These conditions are used in [29] with $\mathcal{X}=1=\mathcal{Y}$ and $V=1$, which serves as the reference model in section $§ 5.2$. Since the temperature equation is excluded from the system, we use the relationship $R e^{2}=R a / P r$, and hence, $R e$ is the only dimensionless parameter that governs the flow.

\subsubsection{Conditions and parameters for a thermally driven flow}

The set of equations (1-3) with $\gamma=1$ governs a thermally-driven, two-dimensional natural convection flow in a cavity: $\bar{\Omega}=\Omega \cup \partial \Omega$. The initial and boundary conditions for the velocity are given by (4) with $V=0$, and that for the temperature field are given by

$$
\begin{array}{ll}
\text { (initial condition) } & \\
\theta=0 & \forall(x, y) \in(0, \mathcal{X}) \times(0, \mathcal{Y}) \text { at } t=0, \\
\text { (boundary conditions) } & \\
\theta=\theta_{0} & \forall y \in[0, \mathcal{Y}], x=0, \text { at } t \geq 0 \text { (left wall), } \\
\theta=\theta_{1} & \forall y \in[0, \mathcal{Y}], x=\mathcal{X}, \text { at } t \geq 0 \text { (right wall), and } \\
\frac{\partial \theta}{\partial y}=0 & \forall x \in[0, \mathcal{X}], y=0, y=\mathcal{Y}, \text { at } t \geq 0 \text { (top \& bottom walls). }
\end{array}
$$

These conditions are used in [30] with $\theta_{0}=0.5, \theta_{1}=-0.5, \operatorname{Pr}=0.71$, and $10^{3} \leq R a \leq 10^{5}$. 


\subsection{Temporal integration}

A fractional step time marching method - also known as the projection method - was proposed in [31] for solving eqs. (1-2), where at each time step an auxiliary or intermediate velocity is obtained from (2) and is updated such that eq.(1) is satisfied. In ref. [31], the projection method was implemented on a collocated or regular grid. Alternatively, the method of Harlow \& Welch (1965) [32] - also known as the MAC method - is a commonly used algorithm in CFD applications that employs a staggered grid. Using the Chorin's projection method (CPM) [31], a fully implicit, second order time integration scheme for (1-3) takes the following form

$$
\begin{gathered}
\boldsymbol{\nabla} \cdot \boldsymbol{u}^{n+1}=0 \\
\frac{\boldsymbol{u}^{n+1}-\boldsymbol{u}^{n}}{\Delta t}+\frac{1}{2}\left(\boldsymbol{u}^{n+1} \cdot \nabla \cdot \boldsymbol{u}^{n+1}+\boldsymbol{u}^{n} \cdot \nabla \cdot \boldsymbol{u}^{n}\right)=-\nabla P^{n+1}+\sqrt{\frac{P r}{4 R a}} \nabla^{2}\left(\boldsymbol{u}^{n+1}+\boldsymbol{u}^{n}\right)+\frac{\gamma \hat{\boldsymbol{k}}}{2}\left(\theta^{n+1}+\theta^{n}\right) \\
\frac{\theta^{n+1}-\theta^{n}}{\Delta t}+\frac{1}{2}\left(\boldsymbol{u}^{n+1} \cdot \nabla \theta^{n+1}+\boldsymbol{u}^{n} \cdot \nabla \theta^{n}\right)=\sqrt{\frac{1}{4 R a P r}} \nabla^{2}\left(\theta^{n+1}+\theta^{n}\right)
\end{gathered}
$$

In this formulation (6-8), the nonlinear dynamics between the velocity $\boldsymbol{u}$ and the temperature $\theta$ is calculated simultaneously, which requires an efficient iterative method. The most common practice would solve (6-7), in the first stage, for $\boldsymbol{u}^{n+1}$ using either a Newton or Picard type iteration, and then (8), in the second stage, for $\theta^{n+1}$, which becomes a linear system. The present solution method is now outlined.

In the first of the fractional time step, the simultaneous system of PDEs are written, using the symbol $u=[\boldsymbol{u}, \theta]^{T}$, as

$$
-\mathcal{A} \nabla^{2} u+\boldsymbol{u} \cdot \boldsymbol{\nabla} u+\frac{2}{\Delta t} u=\mathcal{A} \nabla^{2} u^{n}-\boldsymbol{u}^{n} \cdot \nabla u^{n}+\frac{2}{\Delta t} u^{n},
$$

where

$$
\mathcal{A}=\left[\begin{array}{cc}
\sqrt{\frac{P r}{R a}} & 0 \\
0 & \sqrt{\frac{1}{P r R a}}
\end{array}\right],
$$

$u=[\boldsymbol{u}, \theta]^{T}$ represents the solution at a fractional time step, and $u^{n}=\left[\boldsymbol{u}^{n}, \theta^{n}\right]^{T}$ represents the solution at the previous time step. The coupled nonlinear system (9) takes the following general form

$$
\mathcal{L}(u)=f
$$


where the nonlinear operator $\mathcal{L}$ and the function $f$ represent the left hand and the right hand side of (9) respectively. The system (10) retains the simultaneous nonlinear dependence of the velocity and temperature within a fractional time step. In [24], a similar fractional time stepping was used, where the velocity $\boldsymbol{u}^{n+1}$ was obtained with a Picard's type linearization, and the temperature was solved after the velocity has been computed, thereby ignoring the non-linearly coupled dynamics. In contrast, the present development proposes a fast numerical methodology for solving the simultaneous system of equations (10).

The time evolution of the temperature field $\theta^{n+1}=\theta$ is obtained from (10). However, the same for the velocity field requires additional step; $\boldsymbol{u}^{n+1}=\boldsymbol{u}-\Delta t \nabla P^{n+1}$ such that $\boldsymbol{\nabla} \cdot \boldsymbol{u}^{n+1}=0$. This step accounts for the effect of the pressure gradient force such that eq. (1) is satisfied at each time step, thereby requiring the solution of a Poisson equation

$$
\nabla^{2} P^{n+1}=\frac{1}{\Delta t} \nabla \cdot \boldsymbol{u}
$$

In the present implementation of the CPM algorithm, the nonlinear system (10) of Helmholtz equations and the elliptic Poisson equation (11) are solved at each time step, where the boundary conditions for (11) are Neumann type: $\nabla P^{n+1} \cdot \hat{n}=\boldsymbol{u} \cdot \hat{n}$, and that for (10) are Dirichlet type. In order to optimize the rate of convergence and the computational cost, we have developed a multi-resolution methodology that is now outlined briefly.

\section{A wavelet based numerical methodology}

Recently, wavelet-based methods have appeared in a number of research areas as a dynamically adaptive numerical method $[33 ; 34 ; 35 ; 36 ; 37 ; 24]$. Wavelets can be classified into two categories. The first-generation wavelets have difficulties in dealing with non-periodic boundary conditions [e.g. see, 38]. However, this limitation has been resolved with the introduction of the second-generation wavelet theory in [39]. The recent developments of wavelet methods for CFD applications have been reviewed in [26]. A second-generation adaptive wavelet collocation method (AWCM) for time dependent PDEs was proposed in [40], which was extended to solve two- and three-dimensional elliptic problems [27]. In [41;33; 42], the 2D vorticity equation was solved in the simultaneous space-time domain, assuming the time variable as if another spatial direction, using the second-generation AWCM. To the best of authors' knowledge, the benefits of 
wavelet-based numerical methods have not been fully realized in the area of heat and mass transfer application. In $[43 ; 24 ; 44 ; 45]$, the incompressible Navier-Stokes equations and the temperature equations were solved using an interpolating wavelet method, where the temperature field and the velocity field were computed in two steps using a Bi-Conjugate Gradient STABilized (BiCGSTAB) algorithm. This algorithm requires the linearization of the nonlinear system, and does not take full advantage of the multi-level proprieties of wavelets. In the following section, the wavelet method for approximating a given function has been presented.

\subsection{The wavelet collocation method}

In the present development, second-generation wavelets are constructed on a $d$-dimensional grid

$$
\mathcal{G}^{j}=\left\{\boldsymbol{x}_{\boldsymbol{k}}^{j} \in \Omega: \boldsymbol{k} \in \mathcal{K}^{j}, j \in J, \boldsymbol{x}_{2 \boldsymbol{k}}^{j-1}=\boldsymbol{x}_{\boldsymbol{k}}^{j}\right\}
$$

using the lifting scheme, e.g. see [46; 27] for details. Here, a function $u(\boldsymbol{x})$ is approximated by

$$
\begin{aligned}
& u_{\epsilon}^{j}(\boldsymbol{x})=\sum_{\boldsymbol{k} \in \mathcal{K}^{j} 0} c_{\boldsymbol{k}}^{j_{0}} \varphi_{\boldsymbol{k}}^{j_{0}}(\boldsymbol{x})+\sum_{l=j_{0}}^{j-1} \sum_{\mu=1}^{2^{d}-1} \sum_{k \in \mathcal{K}^{\mu, l}} d_{\boldsymbol{k}}^{\mu, l} \psi_{\boldsymbol{k}}^{\mu, l}(\boldsymbol{x}), \\
& \frac{\left|d_{k}^{\mu, l}\right|}{\| u_{\epsilon}^{j}||_{2}} \geq \epsilon
\end{aligned}
$$

where $\mathcal{G}^{j_{0}}$ is an arbitrary coarse grid, $\mathcal{G}^{j}$ is the desired fine grid, $\mathcal{K}^{j_{0}}$ and $\mathcal{K}^{\mu, j}$ are sets of indices associated with the grid $\mathcal{G}^{j}$, and $\boldsymbol{d}=\left[c_{\boldsymbol{k}}^{j_{0}}, d_{\boldsymbol{k}}^{\mu, l}\right]\left(j_{0} \leq l \leq j-1,1 \leq \mu \leq 2^{d}-1\right)$ denote the wavelet coefficients at level $j[38 ; 27]$.

Let $\boldsymbol{c}=\left[c_{\boldsymbol{k}}^{j}\right]$ denote the numerical values of the function $u(\boldsymbol{x})$ on a grid $\mathcal{G}^{j}$. In the lifting scheme, $c$ is separated into an even or coarse data associated on the grid $\mathcal{G}^{j-1}$ and a odd or detail data on those grid points of $\mathcal{G}^{j}$ which do not belong to $\mathcal{G}^{j-1}$. Odd values are then predicted from even values, and even values are updated using predicted odd values. This is done recursively, starting from a fine grid $\mathcal{G}^{j}$ until a coarse grid $\mathcal{G}^{j_{0}}$ is reached. The process transforms a given function evaluation $c$ into its wavelet transform $\boldsymbol{d}$. Symbolically, we write $\boldsymbol{d}=\mathbb{W} \boldsymbol{c}$ and $\boldsymbol{c}=\mathbb{W}^{-1} \boldsymbol{d}$, where $\mathbb{W}$ and $\mathbb{W}^{-1}$ are forward and inverse wavelet transform operators. Neither $\mathbb{W}$ nor $\mathbb{W}^{-1}$ are formed explicitly, but these transforms are computing using only $\mathcal{O}(N)$ operations - thanks to the lifting scheme.

When $\mathcal{G}^{j}$ is a uniformly refined dyadic grid, there are a total of $N=\left(2^{j}+1\right)^{d}$ collocation 
points. However, only a fraction of these points are associated with the largest $\mathcal{N}$ wavelet coefficients, $\left|d_{\boldsymbol{k}}^{\mu, j}\right| \geq \epsilon|| u_{\epsilon}^{j_{\epsilon}} \|_{2}$, where eq. (12) provides the best $\mathcal{N}$-term approximation $u_{\epsilon}^{j}(\boldsymbol{x})$. Such an approximation does not oscillate at a frequency or wave number that is larger than $2^{j}$ [34]. In other words, the maximum wave number for the approximation (12) is $2^{j}$, which is same as the maximum wave number for a Fourier spectral collocation method on the grid $\mathcal{G}^{j}$. Hence, the adaptive wavelet approximation (12) retains the same wave number truncation as what a Fourier spectral method would do on the grid $\mathcal{G}^{j}$. If $u(\boldsymbol{x})$ represents a property of a fluid motion that has localized spatial structures, we can have $\mathcal{N} \ll N$, which is one well-known advantage of wavelet based methods over spectral methods. The computational complexity of the present wavelet method, using lifted interpolating bi-orthogonal wavelets, is $\mathcal{O}(\mathcal{N})$, which was verified previously, for example see, [27].

All spatial derivatives in eq. (10) and (11) are calculated using a weighted residual collocation method such that

$$
\int R(x) \delta\left(x-x_{k}^{j}\right) d x=0,
$$

where the residual is defined by $R(x):=\mathcal{L} u(x)-\mathcal{L} u_{\epsilon}^{j}(x)$, and $\delta(x)$ is the dirac delta function [e.g. see 41]. Then, following [41], we have $\mathcal{L} u\left(x_{k}^{j}\right)=\mathbb{D W}^{-1} \boldsymbol{d}$, where $\mathbb{D}$ is the resulting differentiation matrix. First, $\boldsymbol{d}$ is obtained by taking the forward wavelet transform of a given $\boldsymbol{c}$, and then the inverse wavelet transform of $\boldsymbol{d}$ at each level $j$ recursively results into a polynomial representation of $u_{\epsilon}^{j}$, which is differentiated to find derivatives. The computational cost of this approach is approximately equal to that of calculating the wavelet coefficients, where neither $\mathbb{D}$ nor $\mathbb{W}^{-1}$ is explicitly formed, due to the lifting scheme. It can also be shown that the maximum error of calculating $q$-th order derivative of $u(x)$ is $\mathcal{O}\left(\epsilon^{1-q / p}\right)$, where $p$ is the order of the polynomial that is used in computing wavelet transform [e.g. 27; 41].

In the present work, an adaptive mesh is constructed recursively, starting from the coarsest grid $\mathcal{G}^{j_{0}}$, and extending it to the desired finest level $j$ such that only those grid points that are necessary to evaluate the approximation (12) are used for a simulation. A details of the mesh generation process is described in [27]. The system of nonlinear equations (10) and elliptic equation (11) are discretized on the adaptive mesh using a method that has been summarized above, without theoretical details, and the readers are referred to the work of $[27 ; 41]$. In this research, a multiresolution algorithm has been proposed for solving (10) and (11). 


\section{A multi-resolution method for nonlinear system}

A multi-resolution or multi-grid solution method transfers the solution from a fine to a coarse resolution, and vice-versa. The present method employs an adaptive mesh, and the grid transfer process is based on the wavelet transform coefficients $\boldsymbol{d}$.

\subsection{Grid transfer operations}

Using a wavelet transform $\boldsymbol{d}^{j}$ on a grid $\mathcal{G}^{j}$, the fine-to-coarse grid transfer $\mathcal{R}^{j}$ is denoted by

$$
u^{j-1}=\mathcal{R}^{j-1} u^{j}
$$

and defined by

$$
u^{j-1}(\boldsymbol{x})=\sum_{\boldsymbol{k} \in \mathcal{K}^{j_{0}}} c_{\boldsymbol{k}}^{j_{0}} \varphi_{\boldsymbol{k}}^{j_{0}}(\boldsymbol{x})+\sum_{l=j_{0}}^{j-2} \sum_{\mu=1}^{2^{d}-1} \sum_{k \in \mathcal{K}^{\mu, l}} d_{\boldsymbol{k}}^{\mu, l} \psi_{\boldsymbol{k}}^{\mu, l}(\boldsymbol{x}), \quad\left|d_{\boldsymbol{k}}^{\mu, l}\right| \geq \epsilon\left\|u_{\epsilon}^{j}\right\|_{2}
$$

which is obtained by discarding the coefficients $d_{k}^{\mu, j-1}, k \in \mathcal{K}^{\mu, j-1}, \mu=1 \ldots 2^{d}-1$ from eq.(12). Similarly, the coarse-to-fine grid wavelet projection $\mathcal{R}^{j}$ is denoted by

$$
u^{j}=\mathcal{R}^{j} u^{j-1}
$$

which is the exact inverse of (13) in the way that the discarded wavelets are now included to obtain (15). These discarded wavelets may not be available in practice, and following [38, Ch.7], we will use the predict wavelets to define the coarse-to-fine grid transfer process (15) approximately by

$$
u^{j}(\boldsymbol{x})=\sum_{\boldsymbol{k} \in \mathcal{K}^{j} j_{0}} c_{\boldsymbol{k}}^{j_{0}} \varphi_{\boldsymbol{k}}^{j_{0}}(\boldsymbol{x})+\sum_{l=j_{0}}^{j-2} \sum_{\mu=1}^{2^{d}-1} \sum_{k \in \mathcal{K}^{\mu, l}} d_{\boldsymbol{k}}^{\mu, l} \psi_{\boldsymbol{k}}^{\mu, l}(\boldsymbol{x})+\sum_{\mu=1}^{2^{d}-1} \sum_{k \in \mathcal{K}^{\mu, j-1}} \tilde{d}_{\boldsymbol{k}}^{\mu, j-1} \psi_{\boldsymbol{k}}^{\mu, j-1}(\boldsymbol{x}),
$$

where $\tilde{d}_{\boldsymbol{k}}^{\mu, j-1}$ are approximations to discarded wavelet coefficients.

Note that the classical multigrid algorithm for a linear elliptic PDE employs either a trivial restriction or a weighted restriction on a uniformly refined grid. A trivial restriction implies that $\left[\mathcal{R}^{j-1} u^{j}\right]_{2 k}^{j-1}=u_{k}^{j}$ because the multi-level grids are nested $\mathcal{G}^{j-1} \subset \mathcal{G}^{j}$ such that $x_{2 k}^{j-1}=x_{k}^{j}$. 
A weighted mean of neighboring values is used to construct a weighted restriction, where the choice of weights is open, but one may consider that the restriction is an adjoint mapping of the prolongation, e.g. see [47]. It is a common practice in multigrid theory that weighted means are used to construct restriction and prolongation operators, but their construction needs special treatment if an adaptive mesh is used.

The present development differs from a classical multi-grid algorithm, and implements the grid transfer operations on an adaptive mesh, using lifted interpolating wavelet transform, where interpolating polynomials of order $\tilde{p}$ and that of order $p$ are used for coarse-to-fine and fine-tocoarse transfers respectively. In all numerical verifications, we have used $p=\tilde{p}=6$. According to the approximation theory, the wavelet transform provides with an accurate represent of a function at coarse or fine resolution, which provides a more appropriate construction for these grid transfer operations rather than using an $a d-h o c$ weighted mean that is commonly in multi-grid theory.

\subsection{A multi-resolution algorithm}

Let $\mathcal{L}\left(u^{j}\right)$ denote the approximation of $\mathcal{L}(u)$ on the grid $\mathcal{G}^{j}$, where $\mathcal{L}$ is a nonlinear advectiondiffusion operator - such as the left hand side of (10), and let us write the discrete form

$$
\mathcal{L}\left(u^{j}\right)=f^{j}
$$

where $f^{j}$ is an approximation to the right hand side of eq. (10). An easier problem

$$
\mathcal{L}\left(u^{j-1}\right)=\mathbf{g}^{j-1}
$$

at a coarser resolution, i.e. on the grid $\mathcal{G}^{j-1}$, is now solved with appropriate definition of $\mathrm{g}^{j-1}$. The current fine resolution approximation $u^{k, j}$ is updated by

$$
u^{k+1, j}=u^{k, j}+\mathcal{R}^{j} \underbrace{\left(u^{j-1}-\mathcal{R}^{j-1} u^{k, j}\right)}_{\text {error }},
$$

where the error is calculated at the coarser resolution, and is transferred to the fine resolution. Note, the calculation of error at a coarser resolution is a key point in saving CPU time. The process is continued until the residual

$$
\mathbf{r}^{j}=f^{j}-\mathcal{L}^{j}\left(u^{k, j}\right)
$$


is minimized by a given tolerance. The right hand side of eq. (18), $\mathbf{g}^{j-1}$, can be formed by transferring the current residual, $\mathbf{r}^{j}$, and the approximate solution, $u^{k, j}$, to the coarser resolution according to

$$
\mathbf{g}^{j-1}=\mathcal{R}^{j-1} \mathbf{r}^{j}+\mathcal{L}\left(\mathcal{R}^{j-1} u^{k, j}\right)
$$

If $\mathbf{g}^{j-1}$ is formed this way, a uniformly refined grid is used, and weighted means are used to construct $\mathcal{R}^{j}$, this multi-resolution algorithm (MRA) takes the similar form of a multi-grid fullapproximation scheme - as described in [48]. However, such a classical multigrid full approximation scheme is not optimal for solving the advection-diffusion problem (17) because a substantial amount of computational work is needed for improving the rate of congvergence, e.g., using anisotropic coarsening and refinement. In the present development, the adaptive mesh and wavelet transform as well as the following development are novel contribution of this MRA with respect to multi-grid theory, and anisotropic coarening/refinement has not been used.

In order to improve the rate of convergence for the above MRA, a process - known as relaxation or smoothing - can be employed to improve the approximation $u^{k, j}$ before transferring to the coarser resolution, as well as to improve $u^{k+1, j}$. A relaxation method aims to remove high frequency oscillation of the error from an approximate solution. A goal of the present work is the development of an efficient relaxation method for heat and mass transfer applications, where a nonlinear advection-diffusion problem is solved. The rate of convergence of the present MRA solver depends also on the relaxation method that solves eq.(17) approximately [e.g., 28].

To see how a relaxation method for a nonlinear problem introduces a high computational overhead, we can re-write eq. (17) in the following compact form:

$$
\mathbf{f}^{j}(\mathbf{u})=0
$$

Since this is a nonlinear system, an improved approximate solution $\mathbf{u}^{k, j}+\mathbf{s}_{k}$ is obtained by solving the linear problem $\mathcal{J}\left(\mathbf{u}^{k, j}\right) \mathbf{s}_{k}=-\mathbf{f}^{j}\left(\mathbf{u}^{k, j}\right)$ approximately, where $\mathcal{J}$ is the Jacobian of the nonlinear system (20) and $\mathbf{s}_{k}$ is the $\mathcal{N} \times 1$ error vector that can be thought as a search direction. In addition to the construction of $\mathcal{J}$, a numerical construction of $\mathcal{J}\left(\mathbf{u}^{k, j}\right) \mathbf{s}_{k}$ is a matrix-vector product, which has a computational cost that scales like $\mathcal{O}\left(\mathcal{N}^{2}\right)$, where $\mathcal{N}$ is the number of grid points. Clearly, the computational overhead is extreme even with an adaptive mesh technique. The solution procedure will be benefited greatly if one computes $\mathcal{J}\left(\mathbf{u}^{k, j}\right) \mathbf{s}_{k}$ using $\mathcal{O}(\mathcal{N})$ complexity as well as finds a 
search direction $\mathbf{s}_{k}$ such that the residual (19) is reduced by a significant amount.

In order to reduce the $\mathcal{O}\left(\mathcal{N}^{2}\right)$ complexity to $\mathcal{O}(\mathcal{N})$, let us consider the Frechet derivative of $\mathbf{f}^{j}(\mathbf{u})$, which is a fundamental Applied Mathematical technique. According to the mathematical definition of the Frechet derivative, we can approximate the action of the Jacobian $\mathcal{J}$ along the search direction $\mathbf{s}_{k}$ in the form of a matrix-vector product, such that

$$
\mathcal{J}\left(\mathbf{u}^{k, j}\right) \mathbf{s}_{k} \approx \frac{\mathbf{f}^{j}\left(\mathbf{u}^{k, j}+\eta \mathbf{s}_{k}\right)-\mathbf{f}^{j}\left(\mathbf{u}^{k, j}\right)}{\eta}
$$

for some small real number $\eta$ [25]. Clearly, the right hand side of (21) can be evaluated with $\mathcal{O}(\mathcal{N})$ complexity when the cost of computing $\mathbf{f}^{j}$ scales like $\mathcal{O}(\mathcal{N})$. Hence, in the above development, the problem is linearized approximately with $\mathcal{O}(\mathcal{N})$ complexity.

Let us now develop a line search method to relax (17) i.e. to reduce non-smooth error from a given approximate solution $\mathbf{u}^{k, j}$ such that $\left\|\mathbf{f}^{j}\left(\mathbf{u}^{k, j}\right)\right\|_{2}$ is minimized by some factor. Most line search algorithms require to find the search direction $\mathbf{s}_{k}$ to be a descent direction, satisfying

$$
\left\|\mathbf{f}^{j}\left(\mathbf{u}^{k, j}+\alpha_{k} \mathbf{s}_{k}\right)\right\|_{2}<\left\|\mathbf{f}^{j}\left(\mathbf{u}^{k, j}\right)\right\|_{2}
$$

where the positive scalar $\alpha_{k}$ is the step length. In the present development, the vector $\mathbf{s}_{k}$ is computed from the linear combination of $m$ independent vectors, and hence, without loss of generality, we can normalize the step length $\alpha_{k}=1$. Here, $\mathbf{s}_{k}$ can be determined, using a Krylov subspace, $\mathcal{K}_{m}\left(\mathcal{J}, \mathbf{f}^{j}\left(\mathbf{u}^{k, j}\right)\right)$ such that

$$
\min _{\mathbf{s}_{k} \in \mathcal{K}_{m}}\left\|\mathcal{J}\left(\mathbf{u}^{k, j}\right) \mathbf{s}_{k}+\mathbf{f}\left(\mathbf{u}^{k, j}\right)\right\|
$$

where $m$ is the dimension of the Krylov sub-space $\mathcal{K}_{m}$. For interested readers, we refer to [Ch 7.3 of 48] for a detailed mathematical analysis of this Krylov method.

In the JFNK solver, e.g. see [25], eq. (21) optimizes the computational complexity, and normally, a non-adaptive mesh is used. Moreover, $m$ will be as large as $\mathcal{N}$ unless an appropriate problem dependent pre-conditioning matrix is found, which is a major drawback for the JFNK method. In our development, $m$ is small, usually has a value from 3 to 5 , because the above Krylov method is used only in the relaxation sweep of the proposed multi-resolution algorithm. 


\subsection{The implementation on an adaptive mesh}

According to eq. (12), if an intermittent function $u(\boldsymbol{x})$ is sampled on a grid $\mathcal{G}^{j}$, we can represent this function using only a fraction of the wavelet coefficients $\boldsymbol{d}^{j}$. In practice, solving a transient problem on the grid $\mathcal{G}^{j}$ and using the wavelet transform of this solution do not bring any advantage to simulating a flow. Instead, we need to develop an algorithm that finds the fraction of the coefficients, i.e. the fraction of the grid $\mathcal{G}^{j}$ without calculating the flow on the entire grid. For this reason, one cannot apply the wavelet method directly to simulate a fluid flow.

Let us now summarize the implementation of the proposed MRA on an adaptive mesh that is obtained recursively starting from a given coarse grid $\mathcal{G}^{j_{0}}$, and updating it dynamically as the flow exhibits localized features.

1. Start with $u^{k, j}$ for the solution of (17) on the present level $j$, and check if $\left\|\mathbf{r}^{j}\right\|_{2}<$ tolerance. (Here, $u^{k, j}$ may be the initial guess at the begining of the process.) If the tolerance is satisfied, we have a solution, and continue onto step 3 to test whether the mesh is sufficient.

2. Perform $\gamma$ steps of MRA iterations on the current level. The choice of the parameter $\gamma=1$ results in a $\mathrm{V}$-cycle and $\gamma=2$ results in a $\mathrm{W}$-cycle iterations (e.g. see, [28] for a details of multi-grid $\mathrm{V}$ - and $\mathrm{W}$-cycle iterations), and go to step 1 .

3. Perform a wavelet transform and analyze the one-to-one mapping between the wavelet coefficients $\boldsymbol{d}=\left[d_{k}^{j}\right]$ and the grid points $\boldsymbol{x}=\left[x_{k}^{j}\right]$. All grid points that are associated with large wavelet coefficients i.e. $\left|d_{k}^{j}\right| /|| u^{j} \|_{2} \geq \epsilon$ are marked for mesh refinement as active points at the present level. Mark all other grid points for deletion or coarsening.

4. If there are no points for refinement, we have a mesh and a solution. Remove all refinement/coarsening flags and stop iteration, otherwise, continue onto the next step.

5. Refine and coarsen the mesh, and construct the next level grid, consider only those collocation points that belong to a suitably defined neighbors of the active grid points at the present level [40].

6. The set of active points and neighbors constitute the next level mesh. Ensure that all active points on the present level and the points on the boundary are included to the next level approximate mesh. This criterion is necessary to ensure that adaptive grids are also nested so that wavelet decomposition and reconstruction are stable. 
7. Use the grid transfer operation (16) to obtain an approximate solution on the next level mesh. Go to step 2.

In the following section, the proposed method is verified by numerical examples.

\section{Numerical experiments}

\subsection{Verification for error and rate of convergence}

In order to demonstrate the accuracy and flexibility of the proposed method, we first consider the numerical solution of the Poisson-Boltzmann equation

$$
-\nabla^{2} u+a \sinh (b u)=f
$$

where $a$ and $b$ are constants. The right hand function $f$ and Dirichlet boundary conditions are chosen such that the solution of (22) is given by

$$
u(x, y)=\alpha_{1} \exp \left(-\left(x^{2}+y^{2}\right) /\left(2 \mu_{0}\right)\right)-0.5 \sin (\pi x) \sin (\pi y)
$$

where $\alpha_{1}=1.0, a=1.0, b=1.0$, and $\mu_{0}=10^{-3}$ have been used. The problem can be considered as a toy model for the purpose of numerical verification. Fig. 1(a) presents the solution of (22) at various resolutions $17 \times 17,33 \times 33,65 \times 65,257 \times 257,513 \times 513$, and $1025 \times 1025$, showing that the solution is converged iteratively if the resolution increases. Fig. 1(b) presents the corresponding adapted grids, verifying that grid points are concentrated near the point $(0,0)$, where the solution has a sharp gradient. These plots in Figs (1(a)-1(b)) demonstrate clearly that the approximation error is reduced, and the mesh is refined only locally if the resolution increases.

For this simulation, the resolution of a uniform grid $\mathcal{G}^{j}$ is given by $\left(m_{x} 2^{j-1}+1\right) \times\left(m_{y} 2^{j-1}+1\right)$, where using $m_{x}=m_{y}=2$, the coarsest grid $\mathcal{G}^{1}$ has a resolution $3 \times 3$ or 9 grid points, the finest grid $\mathcal{G}^{10}$ has a resolution $1025 \times 1025$ or 1050625 grid points, and a tolerance $\epsilon=10^{-4}$ is used to obtain the finest grid $\mathcal{G}^{10}$ according to the algorithm that has been presented in $\S 4.3$. Here only 12473 points of the uniform grid $\mathcal{G}^{10}$ is used for the simulation. The number of points in the adapted grid $\mathcal{G}^{10}$ is 12473 , which is about 84-times less than $1025 \times 1025$ or 1050625 points in the non-adapted grid. Moreover, the number of points 12473 is equivalent to a resolution $112 \times 112$, 
which means that the resolution has been increased by about a factor of 8 or 9 in this case without increasing the global number of grid points. This experiment exhibits clearly the advantage of adaptive mesh refinement approach.

As summarized in table 1, numerical experiments with increasing the resolution each time by a factor of 2 , where the multi-level grids vary from a $17 \times 17$ resolution to $1025 \times 1025$ resolution, indicates that the rate of convergence is independent of the resolution. Using numerical experiments for $10^{-1} \leq \epsilon \leq 10^{-6}$, we have calculated the error $\left\|u(x, y)-u_{\epsilon}^{j}(x, y)\right\|_{2}$ and the number of points $\mathcal{N}$, where $u(x, y)$ stands for the exact solution and $u_{\epsilon}^{j}(x, y)$ stands for the numerical solution for each value of $\epsilon$. Fig. 2(a) shows that the error is $\mathcal{O}(\epsilon)$ and Fig. 2(b) shows that the error is $\mathcal{O}\left(\mathcal{N}^{-3}\right)$. In other words, the tolerance $\epsilon$ controls the error linearly, and a $50 \%$ reduction of the error increases $\mathcal{N}$ by only about 25\%. In Fig. 2(c) the CPU[s] time is plotted against $\mathcal{N}$, which verifies $O(\mathcal{N})$ complexity such that the computational cost scales linearly with the number of computational degrees of freedom $\mathcal{N}$.

The above numerical test verifies the performance of the proposed algorithm.

\subsection{Numerical simulation of a shear-driven flow}

A shear-driven cavity flow, where one wall of the cavity moves at a constant velocity on its own plane, exhibits a boundary layer of thickness, $\delta \propto R e^{-1 / 2}$, and is a classical test problem for the assessment of CFD codes. In order to verify the accuracy of the present model, where a fine mesh is used only in the region of boundary layer, comparison results are summarized in this section. The initial and boundary conditions corresponding to a shear-driven $2 \mathrm{D}$ flow in a cavity: $\bar{\Omega}=\Omega \cup \partial \Omega$ are given by (4).

\subsubsection{Comparison with reference solutions}

In Fig. 3, the simulated velocity $u(0.5, y)$ is compared with data presented in [49] and [29], showing a good agreement between the present and reference solutions. Note that [49] used a Chebyshev collocation method, employing 25600 grid points at $R e=1000$, and [29] used a multigrid method on uniformly refined multi-level meshes, employing 65536 grid points at $R e=1000$ (as well as various other values of $R e$ ). Using a tolerance $\epsilon=10^{-3}$, the present model requires only 3416 grid points, which is about $13 \%$ or $5 \%$ of the grid points required by [49] and [29] respectively, but retains an accuracy that is comparable to these reference simulations. For the present simulation, 
the number of grid points also increases to 10275 and 25434 if the tolerance is decreased to $\epsilon=10^{-4}$ and $10^{-6}$ respectively, where the later is about the same as that of [49]. Fig 4(a) compares velocity $u(0.5, y)$ for a range of tolerance values $10^{-2} \leq \epsilon \leq 10^{-6}$, which shows that a tolerance between $10^{-2}$ and $10^{-3}$ is suffincient. This numerical experiment exhibits that the proposed model reduces the computational work units by reducing drastically the number of grid points without introducing significant error in comparison with numerical data from [29; 49].

A principal objective of the present study includes a space and time adaptivity such that the time step, $\Delta t$ is not restricted by the CFL condition. In order to assess the cost and error of the time integration scheme in such an adaptive mesh CFD model, let us define a CFL number by

$$
\mathrm{CFL}_{\max }=\frac{\max (\|u\|) \max (\Delta t)}{\min (\Delta x)}
$$

where $\mathrm{CFL}_{\max }=1$ means that $\max (\Delta t)=\min (\Delta x)$ because $\max (\|u\|)=1$ for this simulation. Since an explicit time integration scheme requires $\mathrm{CFL}_{\max } \leq 1$, the time step, $\Delta t \leq \min (\Delta x)$, will be extremely small if the mesh is refined locally in the region of sharp change of the solution. Here, $\Delta t$ is adapted dynamically so that a given $\mathrm{CFL}_{\max }$ is satisfied. The experiment with various $\mathrm{CFL}_{\max }$ values between 1 and 6 , as presented in Fig. 4(b), shows that the time step $\Delta t$ can be adjusted according to a desired accuracy without being restricted by the CFL number, which is a distinct feature of the proposed model with respect to classical CFD techniques. Fig. 4(b) shows clearly that a 6 times larger CFL number retains the accuracy within the tolerance limit, which is clear from a visual comparison of computed $u(0.5, y)$ between Figs. 3-4.

These comparison tests reveal good agreement with reference solutions as well as confirms the accuracy of the present solution although a sparse grid is used with a large $\Delta t$ and large CFL number.

\subsection{Flow in a differentially heated cavity}

We have now simulated a flow in a differentially heated cavity for two main reasons. First, this is a prototypical problem for verifying a CFD algorithm, which is relevant to many industrial applications. Secondly, the flow includes the gravitational effects, where density variation occurs in the vertical direction due to thermal effect. This flow is either driven by only a thermal gradient - known as the natural convection or driven by both a shear and a thermal gradient - known as 
the mixed convection. Results for a natural convection flow is presented. The characteristic dimensionless parameter is the Rayleigh number, $R a$, where the flow remains steady or laminar if $R a \leq R a_{\text {critical }}$, but transitions to turbulence occur otherwise. This characteristics of the flow makes it a benchmark candidate for understanding the convergence of a new numerical algorithm.

The set of equations (1-3) with $\gamma=1$ governs a thermally-driven, two-dimensional natural convection flow in a cavity: $\bar{\Omega}=\Omega \cup \partial \Omega$. The initial and boundary conditions for the velocity are given by (4) with $V=0$, and that for the temperature field are given by (5). A series of numerical simulations have been performed for $10^{3} \leq R a \leq 10^{9}$ using the same initial and boundary conditions. These numerical simulations agree with the data available from [30].

\subsubsection{Results}

The temperature distributions for $10^{3} \leq R a \leq 10^{9}$ are presented in Fig. 5, where we see that the region of hot or cold fluid is concentrated near the walls as $R a$ increases. In other words, strong temperature gradient occurs near the walls at high $R a$. This development of the thermal boundary layer makes the computation of such a flow a challenging task. As described in [30], a uniform mesh that is sufficient to calculate the flow at $R a=10^{3}$ will become insufficient if $R a$ increases because the narrow boundary layer at high $R a$ requires a fine mesh. Looking at the temperature distribution at $R a=10^{9}$ in Fig. 5, one sees clearly that the mesh needs to be refined locally only near all four walls of the cavity, which is not necessary away from the walls. According to eq. (12) and the algorithm as described in $\S 4.3$, the present MRA identifies the region dynamically, where a large gradient or boundary layer occurs, and determines the numerical resolution that is necessary to resolve such a boundary layer. Our numerical experiments with a tolerance, $\epsilon=5 \times 10^{-3}$, show that the maximum necessary resolution is $128 \times 128$ for $10^{3} \leq R a \leq 10^{7}, 256 \times 256$ for $R a=10^{8}$, and $515 \times 512$ for $R a=10^{9}$. However, at $R a=10^{9}$, the present simulation has used only 11308 points, which is about $4 \%$ compared to the uniform mesh at the resolution $512 \times 512$.

To see the growth of the boundary layer near the side walls, the velocity $v(x, 0.5)$ and the temperature $\theta(x, 0.5)$ are presented in Figs 6(a), 6(b) respectively for increasing values of $R a$. These results are compared with the data presented in [30, e.g. Fig.3], and we see a very good qualitative agreement. The scaling for the velocity field for the present model differs from that for the model of [30]. Hence, a rescaling of the model output shows that the velocity profiles in Fig. 6(a) has a good quantitative agreement with those presented in [30]. 


\subsection{Heat island circulation}

A heat island circulation is a typical horizontal convection driven by the differential surface heating in the atmosphere. In order to include the stratification effect, the temperature field in eq. (3) is decomposed into $\theta(x, y, t)=\theta_{0}+\bar{\theta}(y)+\theta^{\prime}(x, y, t)$, where gravitational force acts along the $y$ direction. This decomposition is equivalent to adding the term $-\frac{1}{F r^{2}} v$ on the right hand side of eq. (3) [10], where the Froude number is defined by $F r=\frac{U}{L \sqrt{\frac{g}{\theta_{0}} \partial \theta} \frac{\partial \theta}{\partial y}}$. Hence, the choice $F r=\infty$ keeps the eq. (3) in its original form, and the choice $F r=1$ makes it equivalent to the model that was presented in [10]. Using this modification to the governing equations, an idealized heat island circulation in a vertical plane has been simulated, where the initial localized heat source at $t=0$ is on the bottom horizontal wall - as shown in Fig.7(a).

The time evolutions at $t=60$ of the initial temperature $\theta(x, y, 0)$ for $R a=10^{3}, 10^{4}$, and $10^{5}$ are presented in Fig. $7(b-d)$. The pattern of the rising plume indicate that the vertical propagation of the plume is reduced if $R a$ is increased, but the plume remains symmetric with respect to the horizontal distance $x$ measured from the center of the heat source. This pattern is a typical characteristic of horizontal convection, which means that the numerical model has simulated a flow that has good qualitative agreement with an actual heat island circulation. In Fig.8(a), we have presented the temperature profile $\theta(x, 0.5,60)$ out of three temperature data presented in Fig. $7(b-d)$, which shows that the maximum temperature along the line $y=0.5$ is reduced when $R a$ is increased. The vertical temperature profiles $\theta(0.5, y, 60)$ in Fig. 8(b) exhibit that the temperature decays rapidly to zero along the vertical line $x=0$, where $(0,0)$ is center of the heat source, and the rate of this decay is faster with higher values of $R a$. This decay is associated with the stratification. To see this let us assume that the temperature field is spatially homogeneous, which simplifies the temperature equation (3) to the form

$$
\frac{\partial \theta}{\partial t}=-\frac{1}{F r^{2}} v
$$

Clearly, the effect of the stratification term is to decay the temperature $\theta$ in the region of positive vertical velocity, $v$. The horizontal profile of the vertical velocity $v(x, 0.5,60)$ in Fig. 8(c) shows that the vertical convection is increasingly localized above the heat source with increasing $R a$. The narrow region of positive $v$ is accompanied by narrow regions of negative $v$, which means that the region where the temperature decays is also accompanied by regions of temperature increase. This explains the wiggly profile for $R a=10^{5}$ in Fig. 8(b). 


\subsubsection{Comparison with reference results}

In [10], an idealized heat islan circulation was investigated numerically, where a fluid that was confined in a 2D region was heated with a localized heat source on the bottom boundary. The numerical simulation in [10] required an extended domain in order to accommodate the horizontally convective circulation. In contrast, the present simulation used a relatively small domain, where horizontal convection is modelled numerically using a Neumann type boundary conditions. When Fig.8(a), 8(b), and Fig.8(c) of the present simulation are compared visually with Figs.10(a), 9(a), and $10(b)$ respectively from the ref. [10], one finds a good agreement despite both simulations are done in different computational domain with different boundary conditions and different numerical techniques. This comparison verifies that our numerical model simulates a heat island circulation as accurate as the data presented in [10].

\section{Summary}

This paper has explored the development of an efficient CFD model for transient heat and mass transfer applications using an adaptive mesh approach. A multi-resolution algorithm has been proposed that explores some of the recent discoveries on advanced computational algorithms. Comprehensive numerical experiments have been conducted for the verification of the algorithm's performance. The numerical accuracy has been verified in two stages. First, a nonlinear mathematical problem has been solved for which the exact solution is known. This verifies the rate of convergence for the iterative method, accuracy of the global numerical solution, and the computational time needed for a high resolution simulation. Second, transient simulations of a shear driven flow, a natural convection, and a heat island circulation have been compared with previously published numerical data. Good quantitative agreements with these data confirm the performance of this novel computational approach.

The proposed MRA algorithm shares the benefits from some advanced techniques that are known to Applied Mathematics and Computational Physics research community. For example, wavelet based techniques provide an efficient method - known as the nonlinear approximation - so that the most significant proportion of the energy under a localized function can be computed using only a small number of grid points without loosing accuracy. To the CFD research community, the need for anisotropic coarsening and refinement has discouraged for not using the full approxima- 
tion scheme. To the Computational Physics research community, the Jacobian-free Newton-Krylov methodology is a powerful algorithm for simulating multi-physics problems, where a problem specific pre-conditioner matrix must be designed for each simulation. Instead of using the FAS and JFNK solver directly, the concept from these algorithms are taken so that a new algorithm can be designed.

The development throughout this research brings novel ideas to scientists whose research interest lie in the numerical simulation of heat and mass transfer problems. The potential future development includes extension to three-dimensional transient problems, for which a parallel version of this code must be developed. This work is currently underway.

\section{Acknowledgments}

JMA and NKRK would like to acknowledge support from NSERC. Partial support for OVV was provided by the National Science Foundation (NSF) under grants ACI-0242457 and CBET0756046 and U.S. Department of Energy under grant No. DE-FG02-07ER64468. Computational facilities were provided by ACEnet, the regional high performance computing consortium for universities in Atlantic Canada. 


\section{References}

1. J.-F. Luo and X. Shen, "Numerical method of the ray tracing-node analyzing method for solving 2-d transient coupled heat transfer in a rectangular semitransparent medium," Numerical Heat Transfer, Part A: Applications, vol. 55, no. 5, pp. 465-486, 2009.

2. A. Sakurai, S. C. Mishra, and S. Maruyama, "Radiation element method coupled with the lattice boltzmann method applied to the analysis of transient conduction and radiation heat transfer problem with heat generation in a participating medium," Numerical Heat Transfer, Part A: Applications, vol. 57, no. 5, pp. 346-368, 2010.

3. L.-C. Wang, M. Su, W.-L. Hu, Z.-M. Lin, L.-B. Wang, and Y. Wang, "The characteristic temperature in the definition of heat transfer coefficient on the fin side surface in tube bank fin heat exchanger," Numerical Heat Transfer, Part A: Applications, vol. 60, no. 10, pp. 848-866, 2011.

4. W.-L. Chen and Y.-C. Yang, "Estimation of the transient heat transfer rate at the boundary of an electronic chip packaging," Numerical Heat Transfer, Part A: Applications, vol. 54, no. 10, pp. 945-961, 2008.

5. M. R. G. Haghighi, M. Eghtesad, and P. Malekzadeh, "A coupled differential quadrature and finite element method for 3-d transient heat transfer analysis of functionally graded thick plates," Numerical Heat Transfer, Part B: Fundamentals, vol. 53, no. 4, pp. 358-373, 2008.

6. M. Sallah and M. T. Attia, "On galerkin technique for transient radiative heat transfer in finite thin media," Numerical Heat Transfer, Part B: Fundamentals, vol. 56, no. 4, pp. 323-334, 2009.

7. A. Narasimhan, K. K. Jha, and L. Gopal, "Transient simulations of heat transfer in human eye undergoing laser surgery," International Journal of Heat and Mass Transfer, vol. 53, no. 1-3, pp. $482-490,2010$.

8. A. Ghazy and D. J. Bergstrom, "Numerical simulation of transient heat transfer in a protective clothing system during a flash fire exposure," Numerical Heat Transfer, Part A: Applications, vol. 58, no. 9, pp. 702-724, 2010. 
9. P. Nithiarasu, K. Seetharamu, and T. Sundararajan, "Finite element modelling of flow, heat and mass transfer in fluid saturated porous media," Arch. Comput. Meth. Engng, vol. 9, pp. 3-42, 2002.

10. T. Dubois and R. Touzani, "A numerical study of heat island flows: Stationary solutions," International Journal for Numerical Methods in Fluids, vol. 59, pp. 631-655, 2009.

11. T.-M. Shih, M. Arie, and D. Ko, "Literature survey of numerical heat transfer (20002̆0132009): Part ii," Numerical Heat Transfer, Part A: Applications, vol. 60, no. 11-12, pp. 883-1096, 2011.

12. L. Elden, "Numerical solution of the sideways heat equation by difference approximation in time," Inverse Problems, vol. 11, pp. 913-923, 1995.

13. L. Elden and T. Reginska, "Solving the sideways heat equation by a wavelet-galerkin method," Inverse Problems, vol. 13, pp. 1093-1106, 1997.

14. L. Elden, F. Berntsson, and T. Reginska, "Wavelet and fourier methods for solving the sideways heat equation," Inverse Problems, vol. 13, pp. 1093-1106, 1997.

15. M. M. Rahman and J. C. Lallave, "Transient conjugate heat transfer during free liquid jet impingement on a rotating solid disk," Numerical Heat Transfer, Part A: Applications, vol. 55, no. 3, pp. 229-251, 2009.

16. G. El Hitti, M. Nemer, and K. El Khoury, "Reducing cpu time for radiative exchange and transient heat transfer analysis using zone method," Numerical Heat Transfer, Part B: Fundamentals, vol. 56, no. 1, pp. 23-37, 2009.

17. S. V. Patankar, "A calculation procedure for two-dimensional elliptic situations," Numerical Heat Transfer, Part A: Applications, vol. 4, pp. 409-425, Oct. 1981.

18. Z.-G. Wu, J.-Z. Zhang, D.-L. Sun, and W.-Q. Tao, “An efficient solver for the algebraic equations resulting from discretization of the governing equations for fluid flow and heat transfer," Numerical Heat Transfer, Part B: Fundamentals, vol. 56, no. 1, pp. 58-74, 2009.

19. G. De Vahl Davis, "Natural convection of air in a square cavity: A bench mark numerical solution," International Journal for Numerical Methods in Fluids, vol. 3, no. 3, pp. 249-264, 1983. 
20. J. Bell, M. Berger, J. Saltzman, and M. Welcome, "Three-dimensional adaptive mesh refinement for hyperbolic conservation laws," SIAM Journal of Scientific Computing, vol. 15, no. 1, pp. 127-138, 1994.

21. M. Berger and P. Colella, "Local adaptive mesh refinement for shock hydrodynamics," J. Comput. Phys., 1989.

22. M. J. Berger and J. Oliger, "Adaptive mesh refinement for hyperbolic partial differential equations," Journal of Computational Physics, vol. 53, no. 3, pp. 484 - 512, 1984.

23. T. Popiolek and A. Awruch, "An adaptive mesh strategy for transient flows simulations," in Computational Modeling (MCSUL), 2009 Third Southern Conference on, pp. 71 -76, nov. 2009.

24. D. Wirasaet and S. Paolucci, "Adaptive wavelet method for incompressible flows in complex domains," Journal of Fluids Engineering, vol. 127, no. 4, pp. 656-665, 2005.

25. D. A. Knoll and D. E. Keyes, "Jacobian-free newton-krylov methods: a survey of approaches and applications," J. Comput. Phys., vol. 193, no. 2, pp. 357-397, 2004.

26. K. Schneider and O. V. Vasilyev, "Wavelet methods in computational fluid dynamics," Annu. Rev. Fluid Mech., vol. 42, no. 1, pp. 473-503, 2010.

27. O. V. Vasilyev and N.-R. Kevlahan, "An adaptive multilevel wavelet collocation method for elliptic problems.," J. Comput. Phys., vol. 206, pp. 412-431, 2005.

28. P. Wesseling, An Introduction to Multigrid Methods. Chichester: John Wiley \& Sons, 1992.

29. U. Ghia, K. Ghia, and C. Shin, "High-re solutions for incompressible flow using the navierstokes equations and a multigrid method," Journal of Computational Physics, vol. 48, no. 3, pp. 387-411, 1982.

30. D. A. Mayne, A. S. Usmani, and M. Crapper, "h-adaptive finite element solution of high rayleigh number thermally driven cavity problem," International Journal of Numerical Methods for Heat \& Fluid Flow, vol. 10, no. 6, pp. 598 - 615, 2000.

31. A. Chorin, "Numerical solution of navier-stokes equation," Math. Commp., vol. 22, pp. 745$762,1968$. 
32. F. H. Harlow and J. E. Welch, "Numerical calculation of time-dependent viscous incompressible flow of fluid with free surface," Physics of Fluids, vol. 8, no. 12, pp. 2182-2189, 1965.

33. J. Alam, N. K.-R. Kevlahan, and O. Vasilyev, "Simultaneous space-time adaptive solution of nonlinear parabolic differential equations," Journal of Computational Physics, vol. 214, pp. 829-857, 2006.

34. A. Cohen, Numerical Analysis of Wavelet Method. Elsevier, 2003.

35. A. Grossmann and J. Morlet, "Decomposition of hardy functions into square integrable wavelets of constant shape," SIAM J. Math. Anal., vol. 15, no. 4, pp. 723-736, 1984.

36. L. Jameson and T. Miyama, "Wavelet analysis and ocean modeling: A dynamically adaptive numerical method WOFD-AHO," Mon. Wea. Rev., vol. 128, no. 5, pp. 1536-1549, 2000.

37. K. Urban, Wavelets in numerical simulation: problem adapted construction and application. Springer, 2002.

38. E. Bacry, S. Mallat, and G. Papanicolaou, "A wavelet based space-time adaptive numerical method for partial differential equations," Mathematical Modelling and Numerical Analysis, vol. 26, pp. 793-834, 1992.

39. W. Sweldens, The Construction and Application of Wavelets in Numerical Analysis. PhD thesis, University of Belgium, 1995.

40. O. V. Vasilyev and C. Bowman, "Second-generation wavelet collocation method for the solution of partial differential equations.," J. Comput. Phys., vol. 165, pp. 660-693, 2000.

41. J. M. Alam, A space-time adaptive wavelet method for turbulence. $\mathrm{PhD}$ thesis, McMaster University, October 2006.

42. N.-R. Kevlahan, J. Alam, and O. Vasilyev, "Scaling of space-time modes with the Reynolds number in two-dimensional turbulence," J. Fluid Mech., vol. 570, pp. 217-226, 2007.

43. D. Wirasaet and S. Paolucci, "An adaptive wavelet method for the incompressible navierstokes equations in complex domains," ASME Conference Proceedings, vol. 2004, no. 46911, pp. 619-631, 2004. 
44. D. Wirasaet and S. Paolucci, "Application of an adaptive wavelet method to natural-convection flow in a differentially heated cavity," ASME Conference Proceedings, vol. 2005, no. 47330, pp. 499-511, 2005.

45. D. Wirasaet and S. Paolucci, "The application of an adaptive wavelet method to the 3-d naturalconvection flow in a differentially heated cavity," ASME Conference Proceedings, vol. 2006, no. 47861, pp. 581-592, 2006.

46. W. Sweldens, "The lifting scheme: A construction of second generation wavelets," SIAM J. Math. Anal., vol. 29, no. 2, pp. 511-546, 1997.

47. W. Hackbusch, Multigrid methods and applications. New York: Springer-Verlag, 1985.

48. P. Wesseling, Principles of Computational Fluid Dynamics. Springer, 2000.

49. O. Botella and R. Peyret, "Benchmark spectral results on the lid-driven cavity flow," Computers \& Fluids, vol. 27, no. 4, pp. 421 - 433, 1998. 


\begin{tabular}{ccc} 
Grid & \# of MRA iteration & Residual \\
\hline $17 \times 17$ & 19 & $8.06 \times 10^{-9}$ \\
$33 \times 33$ & 23 & $1.66 \times 10^{-8}$ \\
$65 \times 65$ & 23 & $1.27 \times 10^{-8}$ \\
$129 \times 129$ & 22 & $6.66 \times 10^{-9}$ \\
$257 \times 257$ & 22 & $6.32 \times 10^{-9}$ \\
$513 \times 513$ & 22 & $6.24 \times 10^{-9}$ \\
$1025 \times 1025$ & 22 & $6.08 \times 10^{-9}$ \\
\hline
\end{tabular}

Table 1: The rate of convergence of the MRA solver is tested by solving (22). The algorithm takes about the same number of iterations to reduce the residual norm by the same factor, which is independent of the resolution. 


\section{Figure Captions}

1 A sequence of approximate solutions of (22) at various resolutions: $17 \times 17,33 \times$ $33,65 \times 65,257 \times 257,513 \times 513$ and $1025 \times 1025$. (a) We see clearly that the error decreases if the resolution increases. (b) Adapted grids corresponding to the solutions in (a). All points at the resolution $17 \times 17$ are used, but the mesh is refined locally so that only a fraction of the higher resolution grids is used to minimized the error. The solutions as well as the adapted grids at resolutions $513 \times 513$ and $1025 \times 1025$ are almost identical. . . . . . . . . . . . . . . . 31

2 (a) The error remains roughly proportional to $\epsilon$; o- numerical data, - - logarithmic slope for $\mathcal{O}(\epsilon)$. (b) Error as a function of the number of adapted grid points $\mathcal{N} ;$ o-, numerical result; --, logarithmic slope for $\mathcal{O}\left(\mathcal{N}^{-3}\right)(c)$ The cpu time[s] is approximately proportional to $\mathcal{N}$; o-, numerical result; --, logarithmic slope

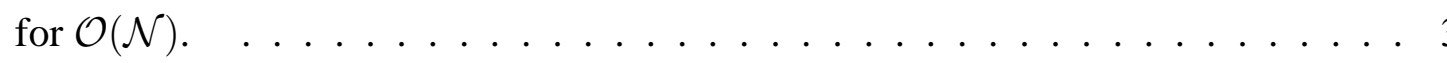

3 The velocity, $u(0.5, y)$, along a line, $x=0.5$, at $R e=1000$ is compared with the data presented in ref. [29] and ref. [49]. Despite the present model uses only a fraction of the grid points compared to reference models, a good agreement agreement in this plot confirms the accuracy of the present model. . . . . . . . . . . 33

4 The effects of varying the tolerance, $\epsilon$, as well as varying the CFL number for computing the velocity, $u(0.5, y)$, at $R e=1000$ are presented, where $u(0.5, y)$ is compared for $(a) 10^{-2} \leq \epsilon \leq 10^{-6}$ and for $(b) 1 \leq \mathrm{CFL} \leq 6$. From a visual comparision between Fig. 3 and Fig. 4 confirms that a tolerance as large as $10^{-2}$ or a CFL number as large as 6 can be used without losing accuracy significantly. . . 34

5 The temperature distributions for various values of the Rayleigh number, $R a=$ $10^{3}, 10^{4}, 10^{5}, 10^{6}, 10^{7}$, and $10^{8}$ at a fixed dimensionless time $T$, when the flow has reached approximately a steady state. The initial temperature at $t=0$ is the same for each case (not shown in the figure). Clearly, a thermal boundary layer is developed as $R a$ increases. (The plot for $R a=10^{8}$ is not shown to optimize the space.) 
6 The development of thermal boundary layers corresponding to temperature distributions in Fig 5 at various values of the Rayleigh number, $10^{3} \leq R a \leq 10^{8}$. (a) Simulated temperature profiles, $\theta(x, 0.5)$, along a fixed line, $y=0.5$ are presented. Only a portion $x \in[0,0.2]$ is shown. Clearly, if $R a$ increases, the width of the boundary layer decreases. (a) Simulated velocity profiles, $v(x, 0.5)$, also exhibit a similar pattern. . . . . . . . . . . . . . 36

7 Time evolutions of an initially localized heat source at $\operatorname{Fr}=1$ and $\operatorname{Ra}=10^{3}$, $10^{4}$ and $10^{5}$ are presented, where the effect of increasing $R a$ on the pattern of the rising plume has been exhibited. The darkest area represents a dimensionless temperature, $\theta=1$ and the lightest area represents $\theta=0 \ldots \ldots$. . . . . . . 37

8 Temperature profiles and velocity profiles corresponding to Fig. 7 for $10^{3} \leq R a \leq$ $10^{5}$ are presented in this figure. (a) Temperature profiles, $\theta(x, 0.5,60)$, along a line $y=0.5$ at $t=60$, show that rising of hot fluid has been suppressed with increasing $R a$. (b) Temperature profiles, $\theta(0, y, 60)$, along the line $x=0$ show that the decay of $\theta$ in $y$ is faster if $R a$ increases. (b) Velocity profile $v(x, 0.5,60)$ along $y=0.5$ show that the maximum of $v$ occurs at $x=0$, which decreases if $R a$ increases. These plots are in good agreement with similar plots presented in [10] . . . . . . . 38 


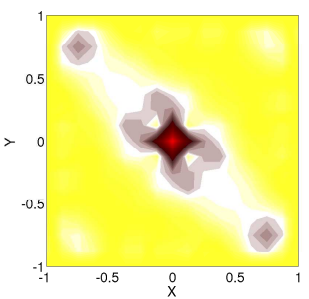

$17 \times 17$

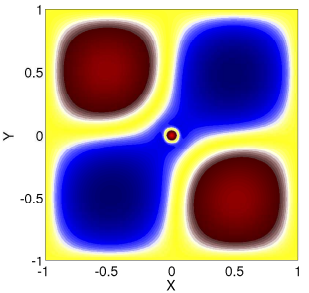

$257 \times 257$

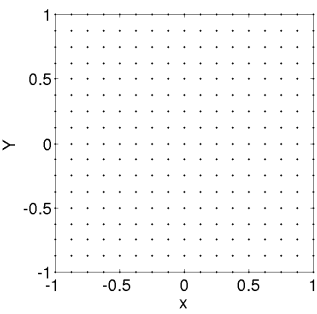

$17 \times 17$

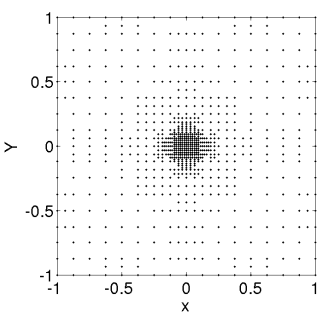

$257 \times 257$

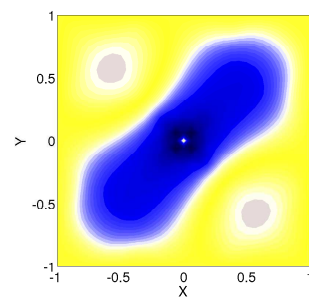

$33 \times 33$

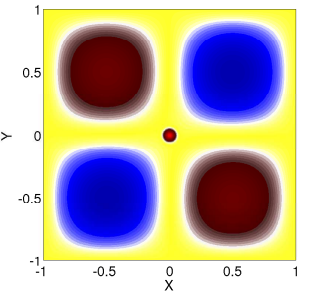

$513 \times 513$

(a)

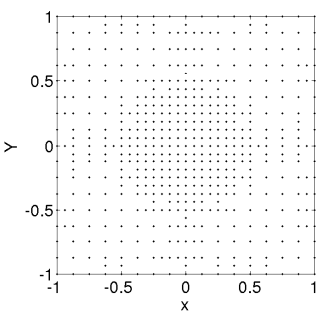

$33 \times 33$

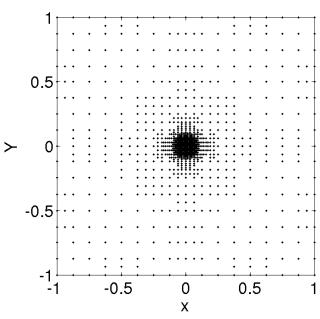

$513 \times 513$

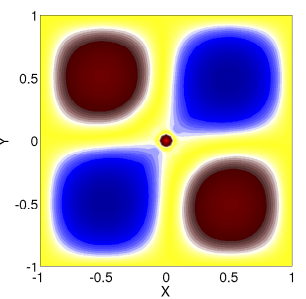

$65 \times 65$

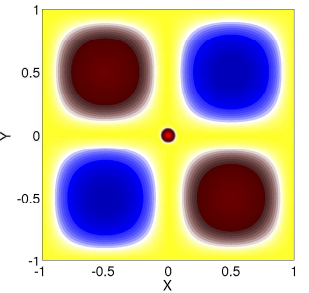

$1025 \times 1025$

(b)

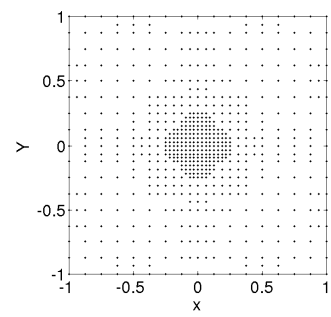

$65 \times 65$

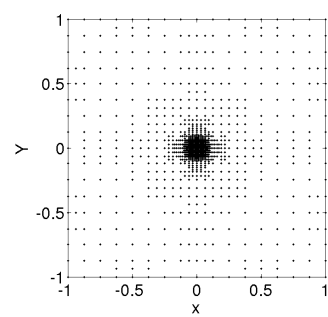

$1025 \times 1025$

Figure 1: A sequence of approximate solutions of (22) at various resolutions: $17 \times 17,33 \times$ $33,65 \times 65,257 \times 257,513 \times 513$ and $1025 \times 1025$. (a) We see clearly that the error decreases if the resolution increases. (b) Adapted grids corresponding to the solutions in (a). All points at the resolution $17 \times 17$ are used, but the mesh is refined locally so that only a fraction of the higher resolution grids is used to minimized the error. The solutions as well as the adapted grids at resolutions $513 \times 513$ and $1025 \times 1025$ are almost identical. 


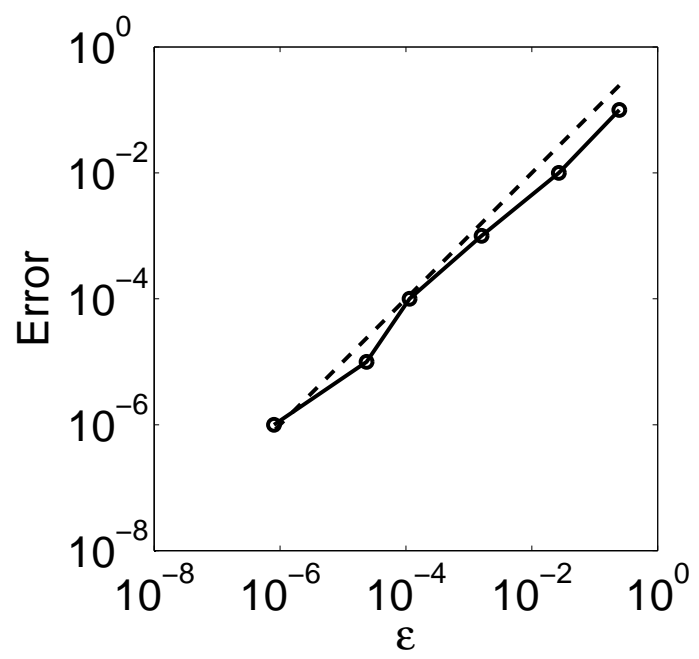

$(a)$

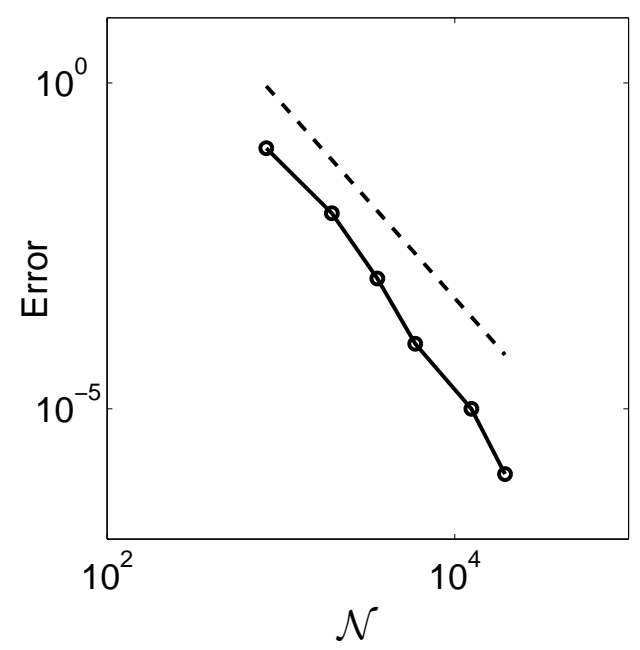

(b)

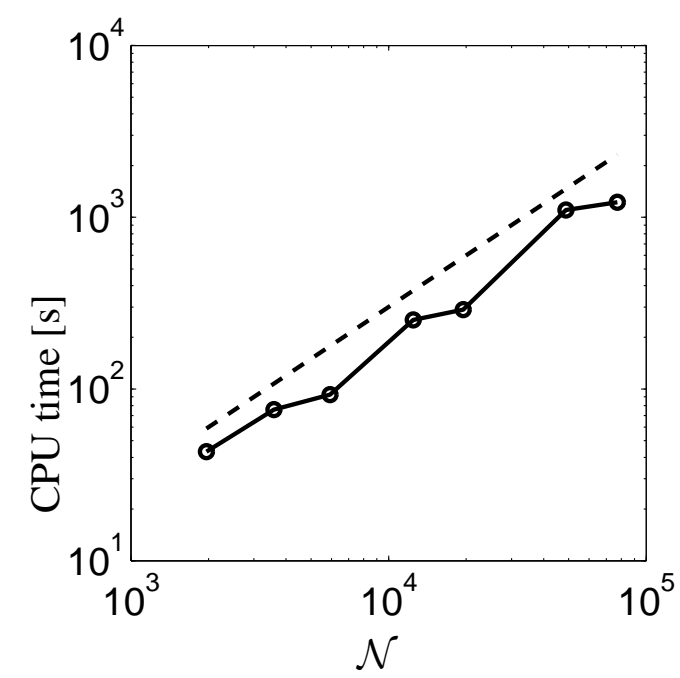

(c)

Figure 2: (a) The error remains roughly proportional to $\epsilon$; o- numerical data, - - logarithmic slope for $\mathcal{O}(\epsilon)$. (b) Error as a function of the number of adapted grid points $\mathcal{N}$; o-, numerical result; --, logarithmic slope for $\mathcal{O}\left(\mathcal{N}^{-3}\right)(c)$ The cpu time[s] is approximately proportional to $\mathcal{N} ;$ o-, numerical result; --, logarithmic slope for $\mathcal{O}(\mathcal{N})$. 


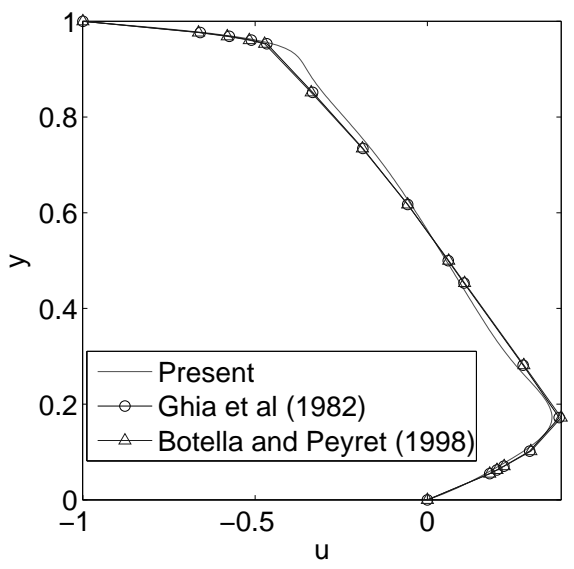

Figure 3: The velocity, $u(0.5, y)$, along a line, $x=0.5$, at $R e=1000$ is compared with the data presented in ref. [29] and ref. [49]. Despite the present model uses only a fraction of the grid points compared to reference models, a good agreement agreement in this plot confirms the accuracy of the present model. 


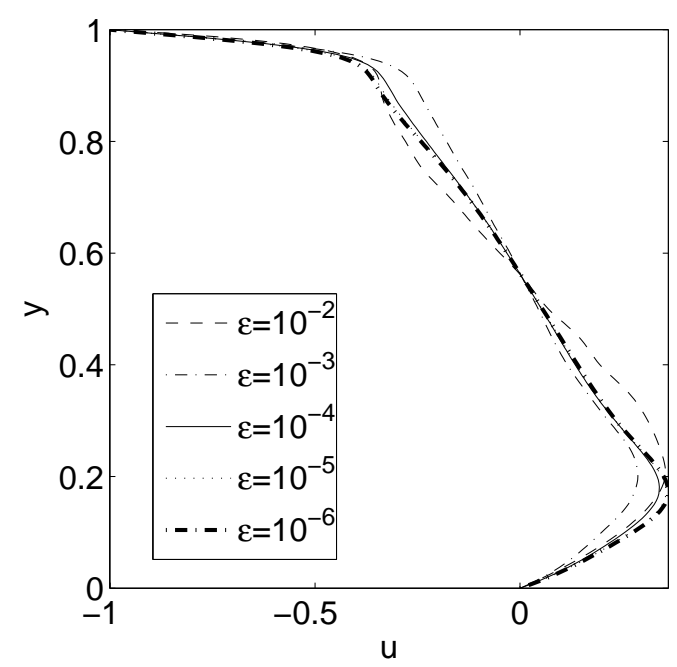

(a)

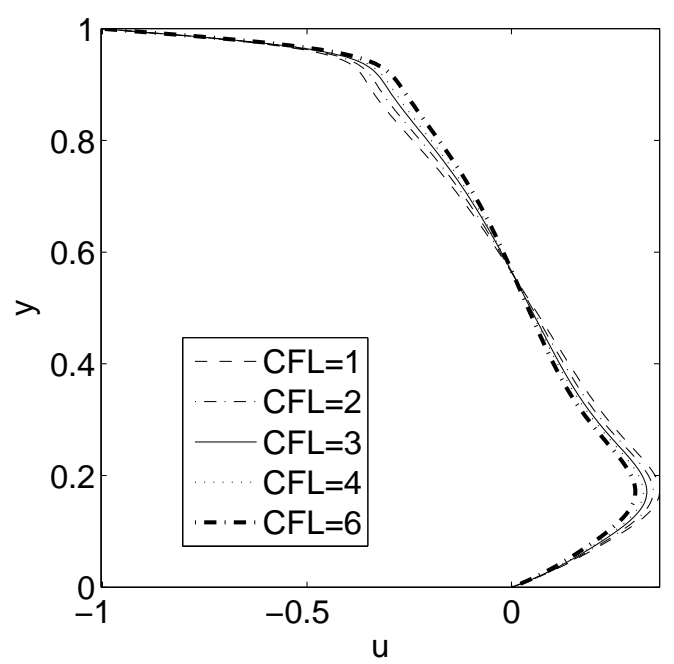

(b)

Figure 4: The effects of varying the tolerance, $\epsilon$, as well as varying the CFL number for computing the velocity, $u(0.5, y)$, at $R e=1000$ are presented, where $u(0.5, y)$ is compared for $(a) 10^{-2} \leq$ $\epsilon \leq 10^{-6}$ and for $(b) 1 \leq \mathrm{CFL} \leq 6$. From a visual comparision between Fig. 3 and Fig. 4 confirms that a tolerance as large as $10^{-2}$ or a CFL number as large as 6 can be used without losing accuracy significantly. 

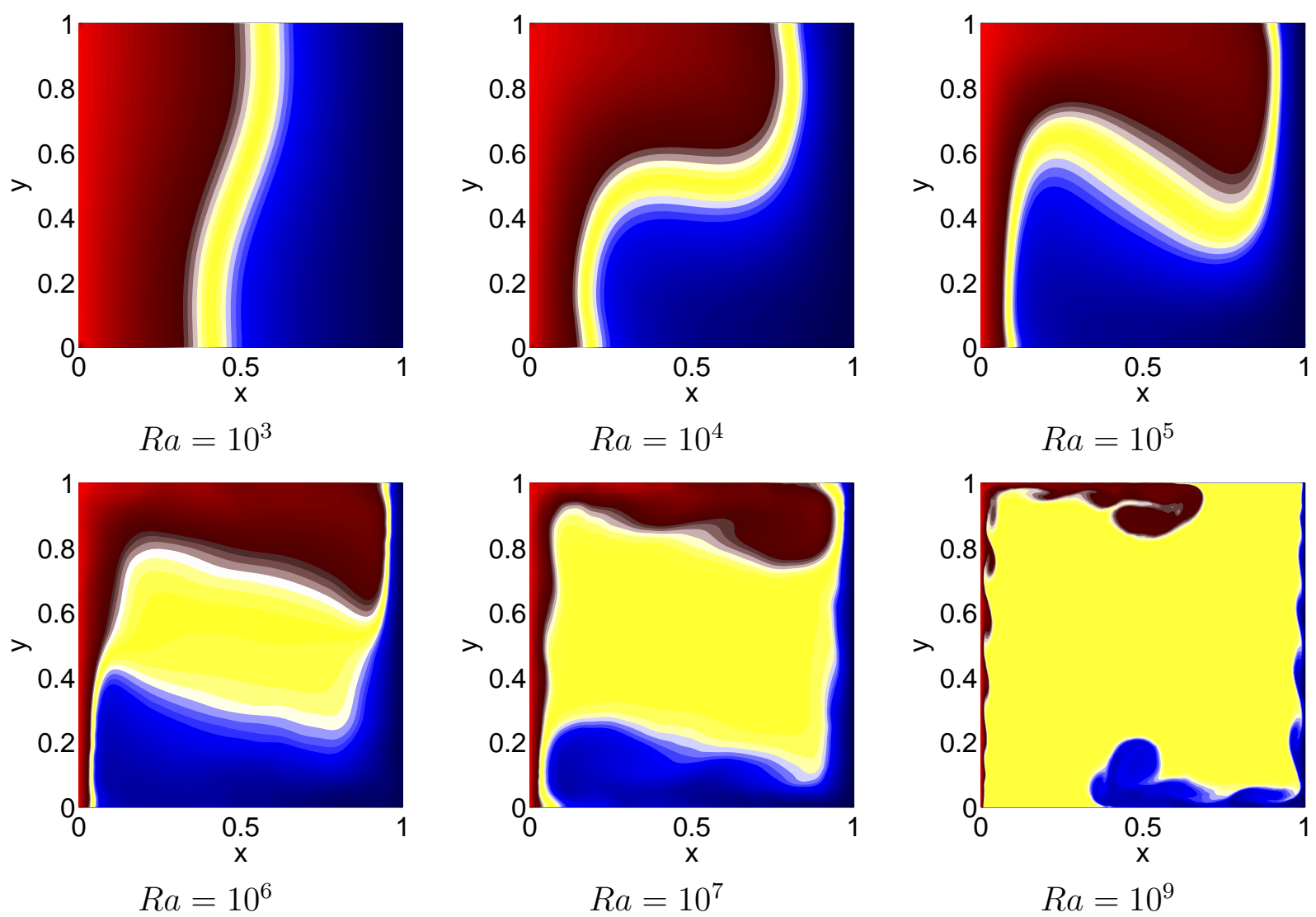

Figure 5: The temperature distributions for various values of the Rayleigh number, $R a=$ $10^{3}, 10^{4}, 10^{5}, 10^{6}, 10^{7}$, and $10^{8}$ at a fixed dimensionless time $T$, when the flow has reached approximately a steady state. The initial temperature at $t=0$ is the same for each case (not shown in the figure). Clearly, a thermal boundary layer is developed as $R a$ increases. (The plot for $R a=10^{8}$ is not shown to optimize the space.) 

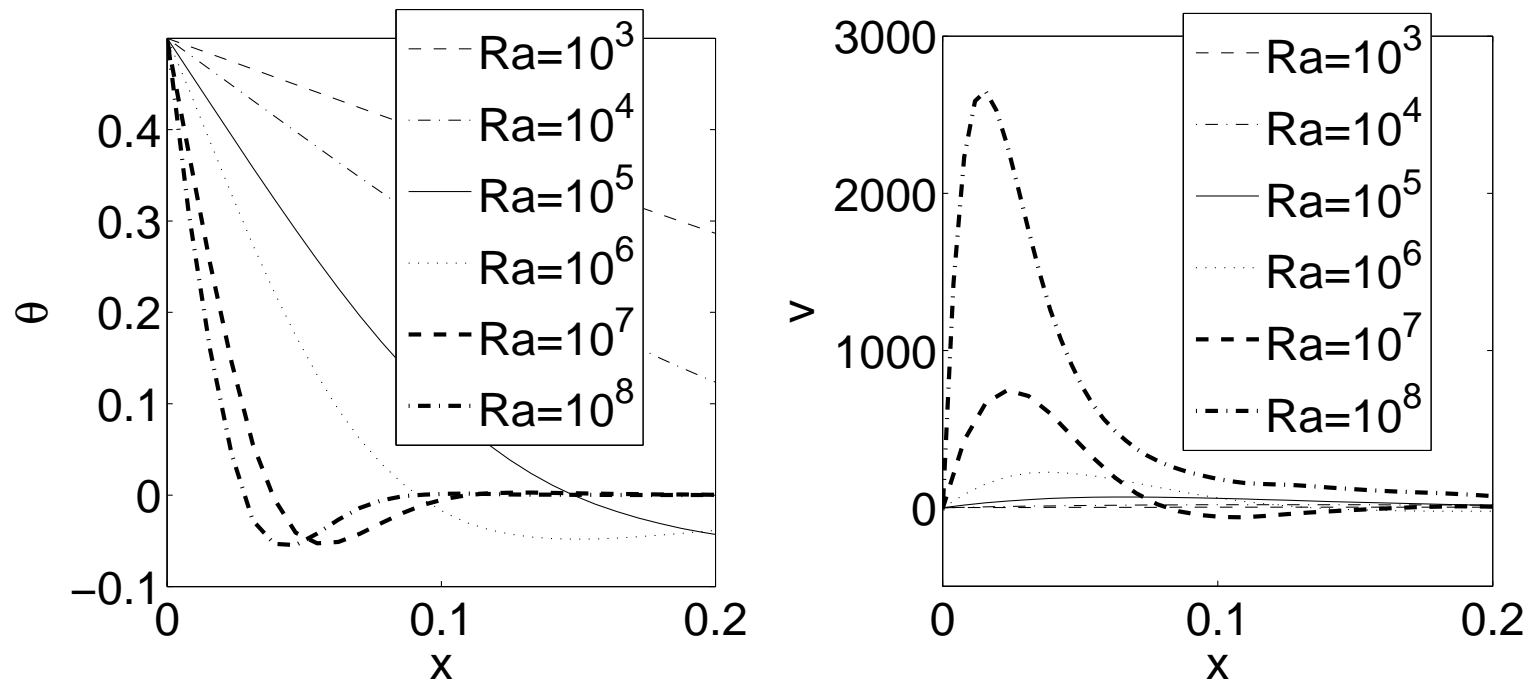

Figure 6: The development of thermal boundary layers corresponding to temperature distributions in Fig 5 at various values of the Rayleigh number, $10^{3} \leq R a \leq 10^{8}$. (a) Simulated temperature profiles, $\theta(x, 0.5)$, along a fixed line, $y=0.5$ are presented. Only a portion $x \in[0,0.2]$ is shown. Clearly, if $R a$ increases, the width of the boundary layer decreases. (a) Simulated velocity profiles, $v(x, 0.5)$, also exhibit a similar pattern. 


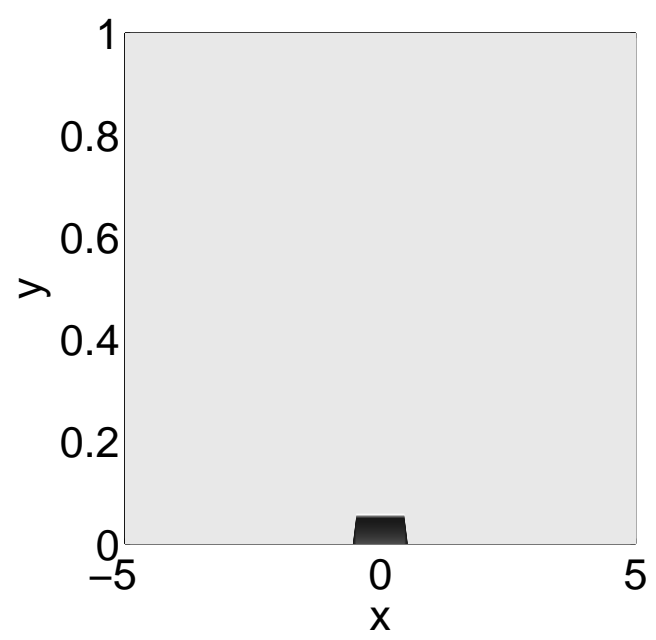

(a) Initial condition

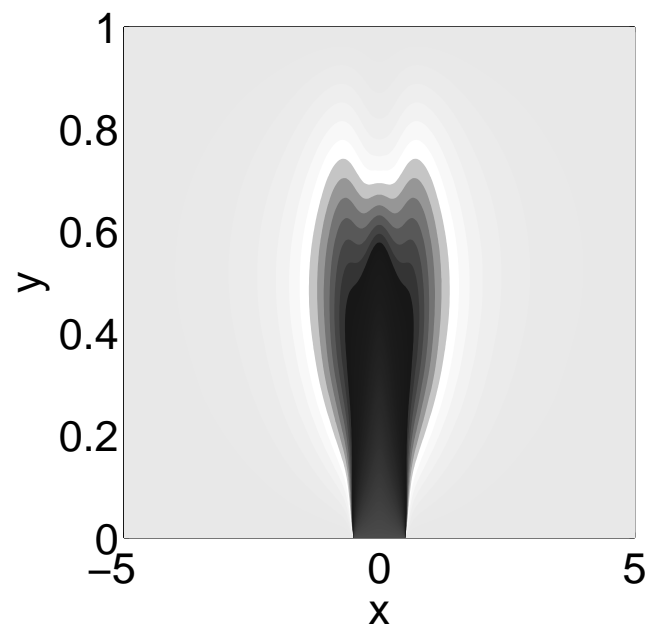

(c) $R a=10^{4}$

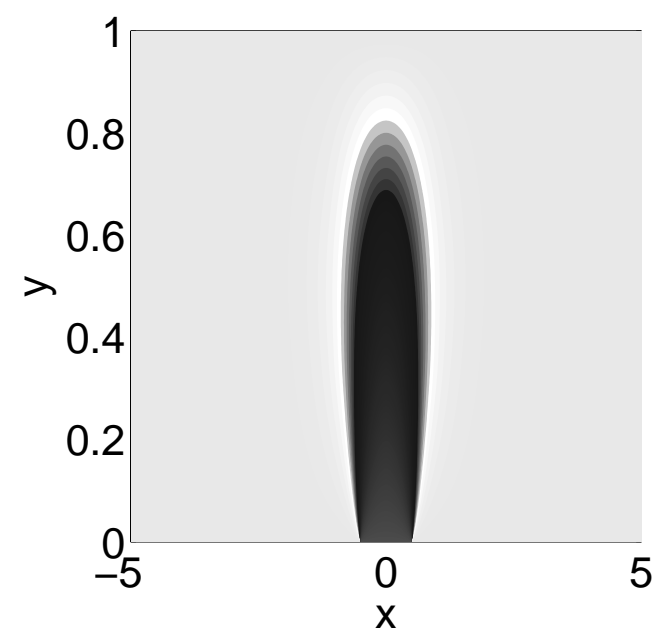

(b) $R a=10^{3}$

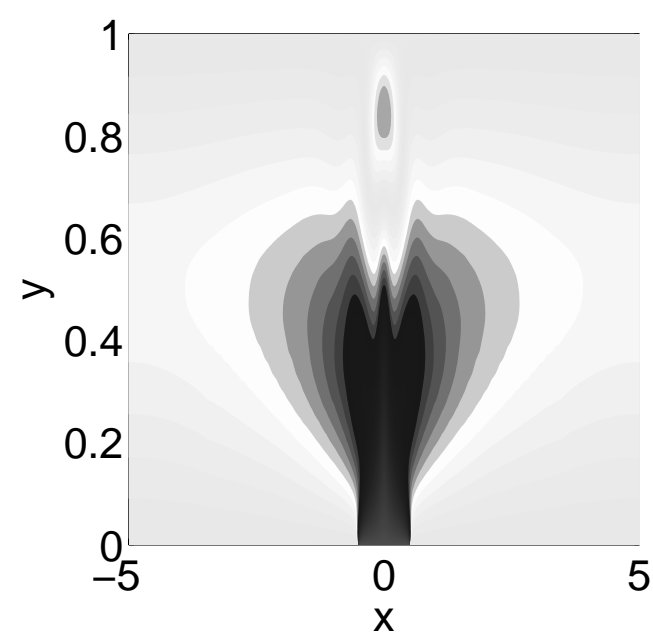

(d) $R a=10^{5}$

Figure 7: Time evolutions of an initially localized heat source at $F r=1$ and $R a=10^{3}, 10^{4}$ and $10^{5}$ are presented, where the effect of increasing $R a$ on the pattern of the rising plume has been exhibited. The darkest area represents a dimensionless temperature, $\theta=1$ and the lightest area represents $\theta=0$. 


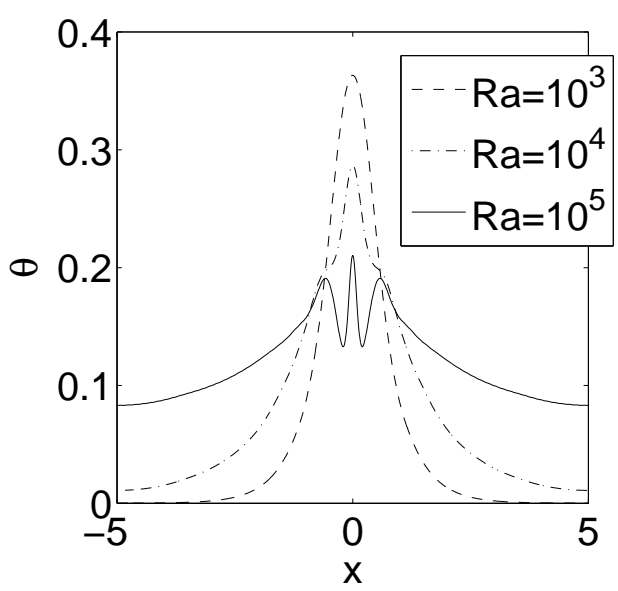

$(a)$

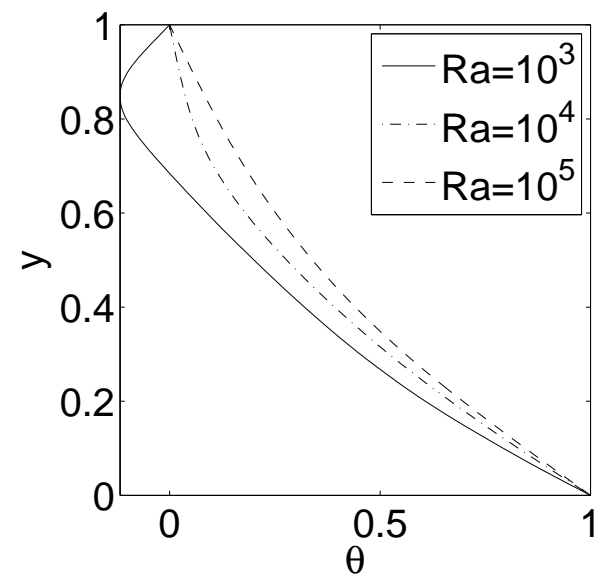

(b)

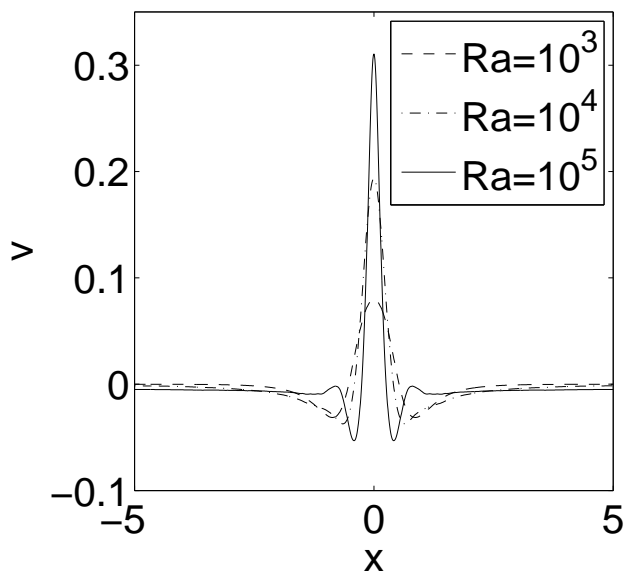

(c)

Figure 8: Temperature profiles and velocity profiles corresponding to Fig. 7 for $10^{3} \leq R a \leq 10^{5}$ are presented in this figure. (a) Temperature profiles, $\theta(x, 0.5,60)$, along a line $y=0.5$ at $t=60$, show that rising of hot fluid has been suppressed with increasing $R a$. (b) Temperature profiles, $\theta(0, y, 60)$, along the line $x=0$ show that the decay of $\theta$ in $y$ is faster if $R a$ increases. (b) Velocity profile $v(x, 0.5,60)$ along $y=0.5$ show that the maximum of $v$ occurs at $x=0$, which decreases if $R a$ increases. These plots are in good agreement with similar plots presented in [10] 